\title{
Dynamics of charge transfer along hydrogen bond*
}

\author{
I.V.Stasyuk, R.Ya.Stetsiv, Yu.V.Sizonenko \\ Institute for Condensed Matter Physics \\ of the National Academy of Sciences of Ukraine, \\ 1 Svientsitskii Str., 79011 Lviv, Ukraine
}

Received September 13, 2002

\begin{abstract}
The pseudospin-electron model is formulated for the description of the correlated proton-electron charge transfer in the complex with hydrogen bonds. The energy spectrum of the model is obtained. The ground state diagram is built. The frequency dependence of the real part of conductivity is calculated. The time evolution of the proton and electron transfer along the hydrogen bond is studied. The time dependences of the mean occupancies of proton positions and electron states are obtained by solving the equations of motion for the density matrix components. The conditions, at which the motion of the proton and electron charges are mutually correlated, are considered.
\end{abstract}

Key words: pseudospin-electron model, proton-electron transfer, hydrogen bond, conductivity

PACS: $72.60 .+g, 36.40 . \mathrm{C}$

\section{Introduction}

The investigation of the microscopic mechanisms of the charge transfer in hydrogen-bonded molecular and crystalline systems and the development of proper models is an urgent problem in the physics of kinetic processes. Usually this phenomenon is assumed to be connected with a diffusive or dynamical type motion of protons. By virtue of the fact that protons are located on hydrogen bonds their transport is caused by the transfer along the bonds (proton tunnelling between equilibrium positions in the double well potential) as well as by the hopping between bonds (which is frequently connected with the ionic groups reorientation). This picture which corresponds to the Grotthuss mechanism was laid at the basis of the recently formulated orientational-tunnelling model of the proton transport ([1], see also [2]).

On the other hand, a series of experimental data [3-7] and the results of quantumchemical calculations $[8,9]$ show the existence of correlation between the proton

${ }^{*}$ This article is dedicated to Prof. Roman Levitskii on the occasion of his 60th birthday. 
displacements and the reconstruction of electron states as well as the change of their occupancy. It turns out that the proton subsystem plays a significant role in the creation of the charge ordered states in crystals with transition metal ions containing hydrogen bonds as elements of structure [4]. The changes in optical spectra formed by electron transitions were detected in BSP and DNP crystals at the redistribution of protons on hydrogen bonds. The available data provide reason enough to make a conclusion about the presence of the significant (in some cases) proton-electron interaction. This interaction can manifest itself as a cooperative proton-electron transfer (PET) [5-7]. The effect of the proton localization on the electron density distribution and on the electron states is clearly seen from the results of quantumchemical calculations (see, for example, [8], where a simple quantum-chemical model has been considered).

The simple model was proposed in [10] for the description of the proton-electron interaction; the proton states on the bonds were represented by means of pseudospin operators. In this model, however, the dynamical part of this interaction, connected with the correlation between the hopping of protons and electron transfer, was not taken into account. This coupling, as it was shown recently [11,12], is significant for the kinetics of ionic and orientational defects in the hydrogen bond network.

The aim of this work is to analyze the microscopic background of the protonelectron interaction, which manifests in particular in the correlated proton-electron hopping. The consideration is performed on the basis of the adiabatic approximation on the example of the single hydrogen bond problem. As a result, the pseudospinelectron model is formulated, in which the generalization of the approach used in [10] is given. The ground energy state of the model is investigated depending on the relations between proton-electron coupling constant $g$, on parameters of effective electron transfer $t$ and on proton tunnelling $\Omega$. The calculations of the complex dynamical conductivity are performed for the investigation of the model dynamics; the frequency dispersion of its real part is analyzed.

The time evolution of the proton and electron transfer along the bond is studied. In particular, the time dependences of the mean occupancies of proton positions and electron states are obtained by solving the equations of motion for the density matrix components. The conditions, at which the motions of the proton and electron charges are mutually correlated, are considered.

\section{Hamiltonian of the linear complex with hydrogen bond. Adi- abatic approximation}

Electronic and vibrational states of A-H-A' linear complex are determined by Schroedinger equation $\hat{H} \Psi=\varepsilon \Psi$, where

$$
H=K_{R}+K_{r}+U(R)+W(R, r) .
$$

Here $K_{R}, K_{r}$ is kinetic energy of ions (nuclei) and electrons; $U(R)$ is potential energy of ions; $W(R, r)$ is energy of the electron subsystem in the field of ions with an account of interaction between electrons. 
In the adiabatic approximation

$$
\Psi(R, r)=\phi(R) \cdot \psi_{R}(r)
$$

the electronic problem has the form:

$$
\left[K_{r}+W(R, r)\right] \psi_{R j}(r)=\lambda_{j}(r) \psi_{R j}(r),
$$

then the ionic (nuclear) problem:

$$
\left[K_{R}+U(R)+\lambda_{j}(R)\right] \phi_{n j}(R)=\varepsilon_{n j} \phi_{n j}(R),
$$

here $j$ are the quantum numbers of electron wave function (numbers of sheets of adiabatic potential); $n$ are the quantum numbers of vibrational states.

Hamiltonian of the electron-ion system in the (2) approximation is of the following form:

$$
\hat{H}=\sum_{n j} \varepsilon_{n j}\left|\phi_{n j}(R) \psi_{R j}(r)\right\rangle\left\langle\phi_{n j}\left(R^{\prime}\right) \psi_{R^{\prime} j}\left(r^{\prime}\right)\right| .
$$

For the adiabatic potential $\lambda_{j}(R)$ with the symmetrical double well

$$
\begin{aligned}
& \phi_{s j}(R)=\frac{1}{\sqrt{2}}\left[\phi_{\alpha j}(R)+\phi_{\beta j}(R)\right], \\
& \phi_{a j}(R)=\frac{1}{\sqrt{2}}\left[\phi_{\alpha j}(R)-\phi_{\beta j}(R)\right],
\end{aligned}
$$

where $\phi_{\alpha, \beta}(R)$ are the functions localized in the potential minima.

Let us introduce the pseudospin representation

$$
\begin{aligned}
\left|\phi_{\alpha j}(R)\right\rangle\left\langle\phi_{\alpha j}\left(R^{\prime}\right)\right| & \rightarrow \frac{1}{2}+S^{z}, \\
\left|\phi_{\beta j}(R)\right\rangle\left\langle\phi_{\beta j}\left(R^{\prime}\right)\right| & \rightarrow \frac{1}{2}-S^{z}, \\
\left|\phi_{\alpha j}(R)\right\rangle\left\langle\phi_{\beta j}\left(R^{\prime}\right)\right| & \rightarrow S^{+}, \\
\left|\phi_{\beta j}(R)\right\rangle\left\langle\phi_{\alpha j}\left(R^{\prime}\right)\right| & \rightarrow S^{-} .
\end{aligned}
$$

Electronic part of (5) expression is formulated in terms of Hubbard operators

$$
\left|\psi_{R_{\alpha} j}\right\rangle\left\langle\psi_{R_{\alpha} j}\left|=X_{\alpha \alpha}^{j j}, \quad\right| \psi_{R_{\alpha} j}\right\rangle\left\langle\psi_{R_{\beta} j}\right|=X_{\alpha \beta}^{j j} .
$$

The $X_{\alpha \beta}^{j j^{\prime}}$ operator transforms the state $j^{\prime}$ of the electron system at the proton localization in $\beta$ well into the state $j$ at the proton localization in $\alpha$ well.

As a result, Hamiltonian (5) is written in the form:

$$
\begin{aligned}
\hat{H}_{\mathrm{eff}}= & \sum_{j} \frac{\varepsilon_{s j}+\varepsilon_{a j}}{2}\left[\left(\frac{1}{2}+S^{z}\right) X_{\alpha \alpha}^{j j}+\left(\frac{1}{2}-S^{z}\right) X_{\beta \beta}^{j j}\right] \\
& +\sum_{j} \frac{\varepsilon_{s j}-\varepsilon_{a j}}{2}\left[S^{+} X_{\alpha \beta}^{j j}+S^{-} X_{\beta \alpha}^{j j}\right]
\end{aligned}
$$


where

$$
\varepsilon_{s j}=\lambda_{j}+\delta_{j}-\frac{1}{2} \Omega_{j}, \quad \varepsilon_{a j}=\lambda_{j}+\delta_{j}+\frac{1}{2} \Omega_{j}, \quad \lambda_{j} \equiv \lambda_{j}\left(R_{\alpha}\right)=\lambda_{j}\left(R_{\beta}\right),
$$

$\delta_{j}$ is a shift of eigenvalue, connected with zero vibration energy; $\Omega_{j}$ is a tunnelling splitting.

The operators of physical quantities in such a representation are rewritten in the following way:

- electronic operator

$$
\hat{A}_{\mathrm{el}}=\sum_{j j^{\prime}}\left[\left\langle\alpha j|\hat{A}| \alpha j^{\prime}\right\rangle\left(\frac{1}{2}+S^{z}\right) X_{\alpha \alpha}^{j j^{\prime}}+\left\langle\beta j|\hat{A}| \beta j^{\prime}\right\rangle\left(\frac{1}{2}-S^{z}\right) X_{\beta \beta}^{j j^{\prime}}\right],
$$

- ionic operator

$$
\begin{aligned}
\hat{A}_{\text {ion }}= & \sum_{j}\left[\langle\alpha|\hat{A}| \alpha\rangle_{j}\left(\frac{1}{2}+S^{z}\right) X_{\alpha \alpha}^{j j}+\langle\beta|\hat{A}| \beta\rangle_{j}\left(\frac{1}{2}-S^{z}\right) X_{\beta \beta}^{j j}\right. \\
& \left.+\langle\alpha|\hat{A}| \beta\rangle_{j} S^{+} X_{\alpha \beta}^{j j}+\langle\beta|\hat{A}| \alpha\rangle_{j} S^{-} X_{\beta \alpha}^{j j}\right] .
\end{aligned}
$$

In the case, when $\langle\alpha|A| \alpha\rangle_{j}=A_{\alpha},\langle\beta|A| \beta\rangle_{j}=A_{\beta}$ and $\langle\alpha|A| \beta\rangle_{j}=0$, we have

$$
\hat{A}_{\text {ion }}=\frac{1}{2}\left(A_{\alpha}+A_{\beta}\right)+\left(A_{\alpha}-A_{\beta}\right) S^{z} .
$$

\section{Pseudospin-electron model of proton-electron transfer of complex with hydrogen bond}

Let us apply the approach described in the item 2 to the simplified case, when only two electron orbitals on the end of the A-H-A' H-bonded cluster are taken into account explicitly. The effective electron transfer

$$
t \sum_{\sigma}\left(a_{1 \sigma}^{+} a_{2 \sigma}+a_{2 \sigma}^{+} a_{1 \sigma}\right)
$$

where $\sigma=\uparrow, \downarrow$, is electron spin, and shift of energy levels due to proton displacement

$$
(\varepsilon+\Delta) \sum_{\sigma} n_{1 \sigma}+(\varepsilon-\Delta) \sum_{\sigma} n_{2 \sigma}
$$

are included into consideration.

It leads to the adiabatic potential in the following form (formed by the lowest electron term)

$$
V(\Delta)=\varepsilon-\sqrt{t^{2}+\Delta^{2}}+\frac{1}{2} K \Delta^{2}
$$

here the last term stands for the elastic strain energy. 
The tunnelling between equilibrium states of proton in the $V(\Delta)$ field is taken into account. Effective Hamiltonian analogous to the (5) can be expressed as:

$$
\begin{aligned}
\hat{H}= & \frac{1}{2}\left(\varepsilon-\Delta_{t}\right) \sum_{\sigma}\left\{n_{1 \sigma}+n_{2 \sigma}-\frac{t}{\Delta_{t}}\left(a_{1 \sigma}^{+} a_{2 \sigma}+a_{2 \sigma}^{+} a_{1 \sigma}\right)\right\} \\
& +\left(\varepsilon-\Delta_{t}\right) \frac{\Delta_{0}}{\Delta_{t}} \sum_{\sigma}\left(n_{2 \sigma}-n_{1 \sigma}\right) S^{Z} \\
& -\frac{\Omega}{2}\left[\left(1-\frac{\Delta_{0}}{\Delta_{t}}\right) a_{1 \sigma}^{+} a_{2 \sigma}+\left(1+\frac{\Delta_{0}}{\Delta_{t}}\right) a_{2 \sigma}^{+} a_{1 \sigma}-\frac{t}{\Delta_{t}}\left(n_{1 \sigma}+n_{2 \sigma}\right)\right] S^{+} \\
& -\frac{\Omega}{2}\left[\left(1+\frac{\Delta_{0}}{\Delta_{t}}\right) a_{1 \sigma}^{+} a_{2 \sigma}+\left(1-\frac{\Delta_{0}}{\Delta_{t}}\right) a_{2 \sigma}^{+} a_{1 \sigma}-\frac{t}{\Delta_{t}}\left(n_{1 \sigma}+n_{2 \sigma}\right)\right] S^{-},
\end{aligned}
$$

here $\Delta_{t}=\sqrt{t^{2}+\Delta_{0}^{2}}, \Delta_{0}$ is defined by the minimum of proton adiabatic potential:

$$
\Delta_{0}^{2}=\frac{1}{K^{2}}-t^{2}
$$

The tunnelling hopping of proton (between minima which correspond to $\pm \Delta_{0}$ values) described by the operators $S^{+}, S^{-}$is correlated in this case with electron transfer from one end of the cluster to another.

\section{Simplified pseudospin-electron model}

In the limit $t \ll \Delta_{0}$, Hamiltonian (16) can be written in the form:

$$
\hat{H}=\sum_{\sigma}\left[E_{0}\left(n_{1 \sigma}+n_{2 \sigma}\right)+g S^{z}\left(n_{2 \sigma}-n_{1 \sigma}\right)-\Omega\left(a_{2 \sigma}^{+} a_{1 \sigma} S^{+}+a_{1 \sigma}^{+} a_{2 \sigma} S^{-}\right)\right],
$$

the parameter $E_{0}=\varepsilon-\Delta_{0} / 2$ hereinafter can play a role of chemical potential $\left(E_{0}=-\mu\right) ; g=\varepsilon-\Delta_{0}$ has a meaning of proton-electron coupling constant.

The second term in (18) describes shift of electron levels at proton displacement (see figure 1); the third term characterizes electron jumps connected with proton tunnelling (figure 2).

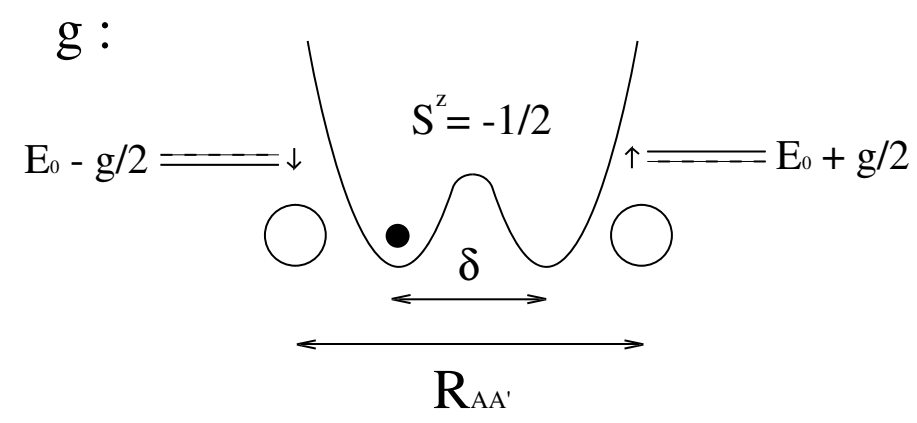

Figure 1. Shift of energy levels caused by proton displacement. 


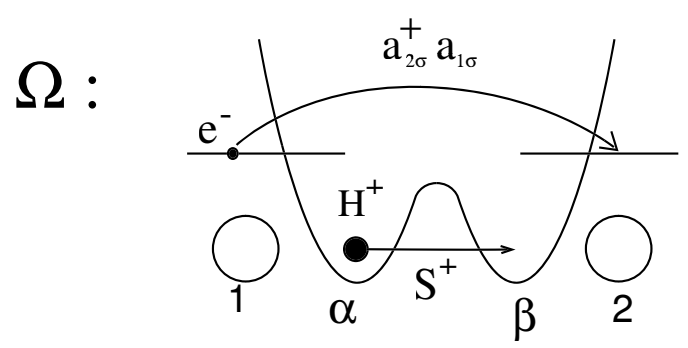

Figure 2. Proton tunnelling correlated with electron transfer.

A model of the (18) type in the case $\Omega=0$ has been introduced before [10] at the description of $\left[\mathrm{M}\left(\mathrm{H}_{2} \mathrm{DAG}\right)(\mathrm{HDAG})\right] \mathrm{TCNQ}, \mathrm{M}=\mathrm{Ni}, \mathrm{Pd}$, Pt crystals, having chainlike sublattice of metal ion and hydrogen bonds as links between them; the proton tunnelling was not taken into account. The model similar to (18) (but without correlated proton-electron tunnelling) is used at the description of locally anharmonic phenomena in high- $\mathrm{T}_{\mathrm{c}}$ superconductors, where the $g S^{z} \sum_{\sigma} n_{i \sigma}$ term characterizes the interaction of conducting electrons with the anharmonic apex oxygen ion subsystem $[13]$.

\section{Numerical values of model parameters}

Numerical values of model parameters were chosen using the experimental data and also based on the results of direct quantum-chemical calculations, performed by us and by other authors. In particular, the value of electron transfer $t$ is determined through some factors. At the increase of hydrogen bond length $R_{00}$, parameter $t$ decreases. The value of $t$ also depends on orbitals involved in electron transfer. In the case of the $\left(p_{\sigma}, p_{\sigma}\right)$ orbitals, which are parallel to the hydrogen bond, the value of transfer integral $t$ is $5-7$ times larger than for $\left(p_{\pi}, p_{\pi}\right)$-orbitals, perpendicular to the hydrogen bond. We obtained this relation using calculations of overlap integrals $\left(p_{\sigma}, p_{\sigma}\right)$ and $\left(p_{\pi}, p_{\pi}\right)$ between $p$-orbitals of oxygen at the ends of hydrogen bonds and considering that parameter $t$ in linear approximation is proportional to overlap integral. At distance $R_{00}=2.40 \AA$ there was obtained $t=0.7 \mathrm{eV}$, i.e., the case of the $\sigma$-coupling; $t=0.12 \mathrm{eV}$, i.e., the case of the $\pi$-coupling.

To evaluate the constant of proton-electron interaction $g$, based on the Hamiltonian of pseudospin-electron model we obtain an expression for the difference between the mean occupations of orbitals on atoms creating the hydrogen bond (or the change of occupations of such atom orbitals at the proton displacement from one equilibrium position to another):

$$
\bar{n}_{2}-\bar{n}_{1}=\frac{g}{\sqrt{g^{2}+4 t^{2}}} \operatorname{th} \frac{\beta}{2} \sqrt{g^{2}+4 t^{2}} .
$$

On the other hand, we evaluate this difference of occupations by a direct quantum-chemical calculation for the cluster with hydrogen bond [8] and obtain $\Delta n=$ 
$\bar{n}_{2}-\bar{n}_{1}=-0.13$; using these data we have, in particular at $t=0.5 \mathrm{eV},|g| \approx$ $0.14 \mathrm{eV}$; at $t=0.05 \mathrm{eV},|g| \approx 0.02 \mathrm{eV}$. Similar calculations were performed in this paper (see appendix) for fragments of crystal structure with hydrogen bonds of KDP-type. We have obtained that difference of occupation of oxygen ions forming hydrogen bond is $\Delta n \approx-0.19$. The charges of oxygen ions are determined by the location of the proton in one of the two possible equilibrium positions and weakly depend on the crystal surroundings or even on the asymmetry of the hydrogen bond (see appendix). Contrary to that, the obtained change of the cluster energy at proton displacement from one equilibrium position on the bond into another, which determine energy of Slater-Takagi configurations, significantly depends on the field of the crystal surroundings of the bond, along which the proton is displaced. The obtained value of energy for configuration with one or three protons (see appendix) is $w=623 \mathrm{~cm}^{-1}$. On the other hand, evaluating this energy as difference of energies of $\mathrm{H}_{n} \mathrm{PO}_{4}$ clusters with different number of protons in the near to current $\mathrm{PO}_{4}$ group equilibrium positions, gives a value $w=54.2 \mathrm{~cm}^{-1}$, that is an order of magnitude smaller than the data known from literature (see $[16,17]$ for example). So, energies of Slater-Takagi configurations are determined not only by the number of protons on each $\mathrm{PO}_{4}$ group in KDP-type crystal as it was considered earlier. To a great extent their values are also caused by the net influence of the surroundings.

The change of orbital occupation of oxygen atoms forming the hydrogen bond, was also calculated by other authors (see, for example, [9]). In $\left(\mathrm{O}_{2} \mathrm{H}_{3}\right)^{-}$case for $p_{\sigma}$ function $\Delta n\left(p_{\sigma}\right)=-0.198$, that corresponds $g<0$; for $p_{\pi}$ functions $\Delta n\left(p_{\pi}\right)=0.102$ $(g>0)$. For complex $\left(\mathrm{O}_{2} \mathrm{H}_{5}\right)^{+}: \Delta n\left(p_{\sigma}\right)=-0.13 \quad(g<0), \Delta n\left(p_{\pi}\right)=0.109 \quad(g>0)$ [9].

Using Hamiltonian (18) we also obtain an expression for potential barrier height for proton on the bond:

$$
h=\sqrt{t^{2}+\Delta_{0}^{2}}-t-\frac{1}{2} K \Delta_{0}^{2} .
$$

On the other hand, potential barrier height was determined from direct quantumchemical calculations [8]. As a result, for the $\Delta_{0}$ parameter, the following values were obtained: $\Delta_{0} \sim 0.01 \cdots 0.2 \mathrm{eV}$ (short bond); $\Delta_{0} \sim 1.0 \cdots 2.0 \mathrm{eV}$ (long bond).

For the energy of tunnelling splitting $\Omega$, calculations performed in [15] give the values $\Omega=0.01 \cdots 0.1 \mathrm{eV}$ depending on the hydrogen bond length.

At $\Omega=0.01 \mathrm{eV}$ value, satisfactory numerical description of the thermodynamical properties of KDP and DKDP-groups of crystals is obtained [16,17].

Electronic and protonic parts of dipole moment of the system with a hydrogen bond are determined correspondingly, by $p$ and $q$ parameters (see (23)). Here $\mu_{e} \equiv$ $p=\frac{1}{2} R_{00} \cdot e, R_{00}=2.40 \AA, \mu_{\mathrm{H}} \equiv q=Z_{\mathrm{H}}^{\mathrm{eff}} \cdot \delta, Z_{\mathrm{H}}^{\mathrm{eff}} \approx 0.25 \mathrm{e}, \delta=0.40 \AA$. 


\section{Dynamical conductivity of hydrogen bond}

Coefficient of dynamical conductivity of the system in accordance with Kubo formula [14] has the following form:

$$
\sigma(\omega)=\int_{0}^{\infty} \mathrm{d} \tau \int_{0}^{\beta} \mathrm{d} \lambda\langle\hat{J}(\tau-\mathrm{i} \hbar \lambda) \hat{J}(0)\rangle,
$$

where $\hat{J}(\tau)$ is the current density operator,

$$
\hat{J}(\tau)=\frac{\mathrm{i}}{\hbar}[\hat{H}, \hat{\mu}] .
$$

The dipole momentum operator $\hat{\mu}$

$$
\hat{\mu}=p \sum_{\sigma}\left(n_{1 \sigma}-n_{2 \sigma}\right)+q S^{z}
$$

includes electron and pseudospin (ion) parts.

Based on the eigenstates of $H_{\text {eff }}$ Hamiltonian $H_{\text {eff }}|r\rangle=E_{r}|r\rangle$, formula (21) can be rewritten as

$$
\begin{aligned}
\sigma(\omega)= & \frac{1}{\hbar^{2}} \sum_{r p}\left(E_{r}-E_{p}\right)\left|\mu_{r p}\right|^{2} \frac{\mathrm{e}^{-\beta E_{p}}-\mathrm{e}^{-\beta E_{r}}}{Z} \\
& \times\left[\pi \delta\left(\omega-\frac{E_{r}-E_{p}}{\hbar}\right)-\mathrm{i} P \frac{1}{\omega-\left(E_{r}-E_{p}\right) / \hbar}\right], \\
Z= & \sum_{a} \mathrm{e}^{-\beta E_{a}} .
\end{aligned}
$$

\section{Time evolution of the proton and electron transfer}

The time dependence of the mean occupancies of electron states $\left\langle n_{1}\right\rangle,\left\langle n_{2}\right\rangle$ and proton positions (or mean values of pseudospin $\left\langle S^{z}\right\rangle$ ) are obtained as follows:

$$
\langle\hat{A}(\tau)\rangle=\operatorname{Sp}(\hat{A} \hat{\tilde{\rho}})=\sum_{r p} A_{r p} \tilde{\rho}_{p r}(\tau)
$$

where $\tilde{\rho}_{p r}(\tau)$ is the time dependent density matrix; the $\hat{A}$ operator can be equal to $n_{1}, n_{2}$ or $S^{z}$.

$$
\tilde{\rho}_{p r}(\tau)=\mathrm{e}^{-\frac{\mathrm{i}}{\hbar}\left(E_{p}-E_{r}\right) \tau} \tilde{\rho}_{p r}(0), \quad \tilde{\rho}_{p r}(0)=\left(\hat{U}^{-1} \rho \hat{U}\right)_{p r}
$$

here $\rho$ is a density matrix for initial state (at time $\tau=0$ ) in the $\left|n_{1 \uparrow}, n_{1 \downarrow}, n_{2 \uparrow}, n_{2 \downarrow}, S^{z}\right\rangle$ representation, $\hat{U}$ is a unitary transformation to the $|r\rangle$ basis of eigenstates of Hamiltonian (16). 


\section{Dynamical properties of hydrogen bond}

Diagonalization of the Hamiltonian (16) was performed using its representation in the matrix form on the $\left|n_{1 \uparrow} n_{1 \downarrow} n_{2 \uparrow} n_{2 \downarrow} ; S^{z}\right\rangle$ basis that includes 32 states. Secular problem of 32 order splits into few independent problems of the lower order, which are determined by the number of electrons on the bond and the total spin value. The ground energy state of the model changes depending on the relation between the values of the $\Omega, t, g$ or $\Delta_{0}$ parameters and also is determined by the position of chemical potential $\mu$ fixed by the electron thermostat, formed by surroundings (with which the complex is in equilibrium). Transformation of the ground state at the change of tunnelling constant is illustrated in figure 3. For some $\mu$, the critical values of $\Omega$ exist, at which the mean number of electrons on the complex is changed, that effects its charge state.

Real part of conductivity $\sigma(\omega)$, calculated using formula (24) has the form of a group of $\delta$-peaks. Their intensities depend on temperature and on the mean number of electrons on the bond; their positions are determined by the values of $g$ (or $\left.\Delta_{0}\right), \Omega$ and $t$. Results of the $\sigma(\omega)$ calculations are presented in Figs. $4-6$. For the case of simplified pseudospin-electron model $(t=0)(18)$ two peaks (maxima) were obtained on the frequency dependence of the $\operatorname{Re} \sigma(\omega)$ function (see figure 4 ). The first of them is connected with the proton tunnelling on the bond, the other one is mostly determined by the constant of the proton-electron interaction $g$. The peaks are shifted to the higher frequency region if the independent electron transfer is present $(t \neq 0)$ and in this case we obtain two groups of peaks; besides that, a new group of peaks appears.

The time dependence of mean occupation of electron states $\left\langle n_{1}\right\rangle,\left\langle n_{2}\right\rangle$ as well as the mean value of pseudospin $\left\langle S^{z}\right\rangle$ (that determines the mean proton position on the bond) are obtained in accordance with formulae (25) and (26). The states with one electron on the bond $|3\rangle=|0100 ; 1 / 2\rangle$ and $|19\rangle=|0100 ;-1 / 2\rangle$ were taken as initial (at $\tau=0)$. At zero value of the transfer parameter $(t=0)$, the time evolution of the mean value of the electron occupation $\left\langle n_{1}\right\rangle$ and the mean value of pseudospin $\left\langle S^{z}\right\rangle$ are synchronized and are described by one harmonic (see figure 7 ) $\hbar \omega_{1}=\Delta E_{1}=0.2 \mathrm{eV}\left(T_{1}=20.8 \cdot 10^{-15} \mathrm{~s}\right)$. A corresponding peak of dynamic conductivity is shown in figure $4 \mathrm{a}, n=1$.

It is obtained, that at $t=0.05 \mathrm{eV}$ allowing for the transitions of the system only from the ground state into the excited ones (they are two in number), the charge transport is described by two harmonics $\hbar \omega_{1}=\Delta E_{1}=0.226 \mathrm{eV}\left(T_{1}=18.2 \cdot 10^{-15} \mathrm{~s}\right)$, $\hbar \omega_{2}=\Delta E_{2}=0.035 \mathrm{eV}\left(T_{2}=120 \cdot 10^{-15} \mathrm{~s}\right.$ ) (see figure 8). As one can see from figure 8 , harmonic $\omega_{2}$ describes synchronized motions in phase of proton and electron, while at frequency $\omega_{1}$ their motion is in antiphase. Two peaks at these frequencies can be observed in the frequency dependence of conductivity (figure $4 \mathrm{c}, n=1$ ). The results of calculations when all possible harmonics are taken into account are presented in figure 9 . The similar results at the other values of model parameters are presented in figure 10 . 


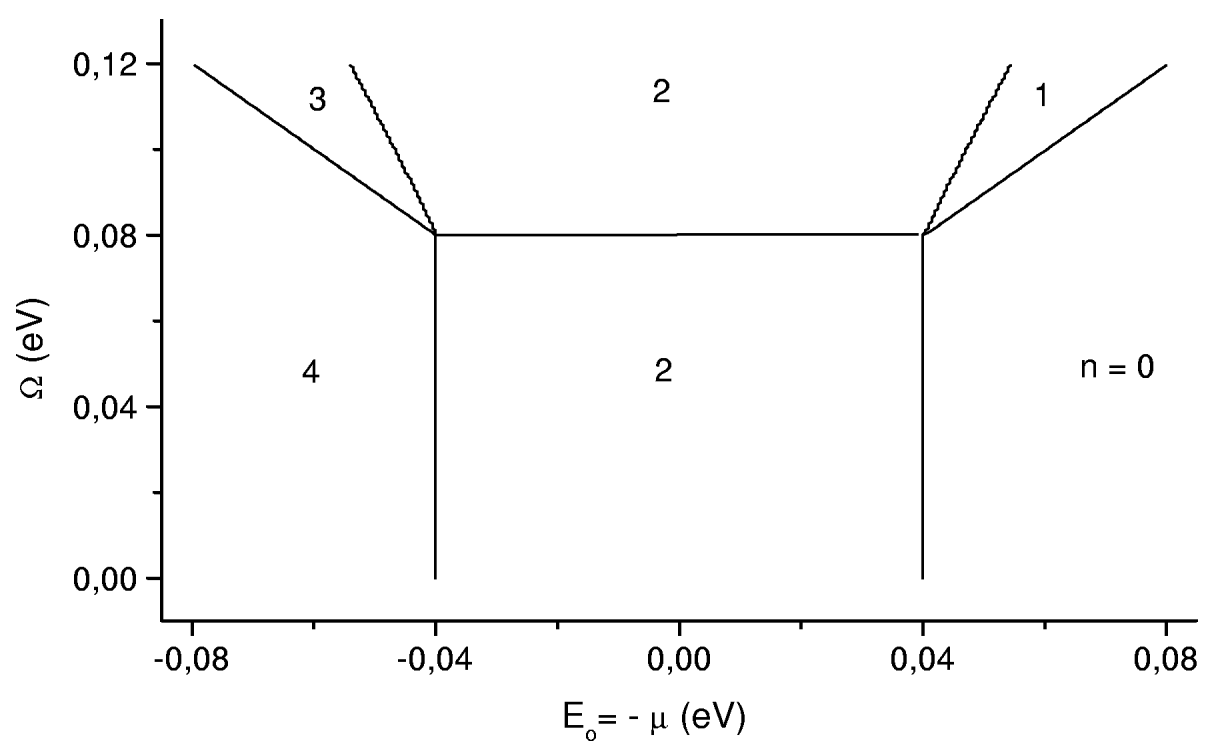

(a)

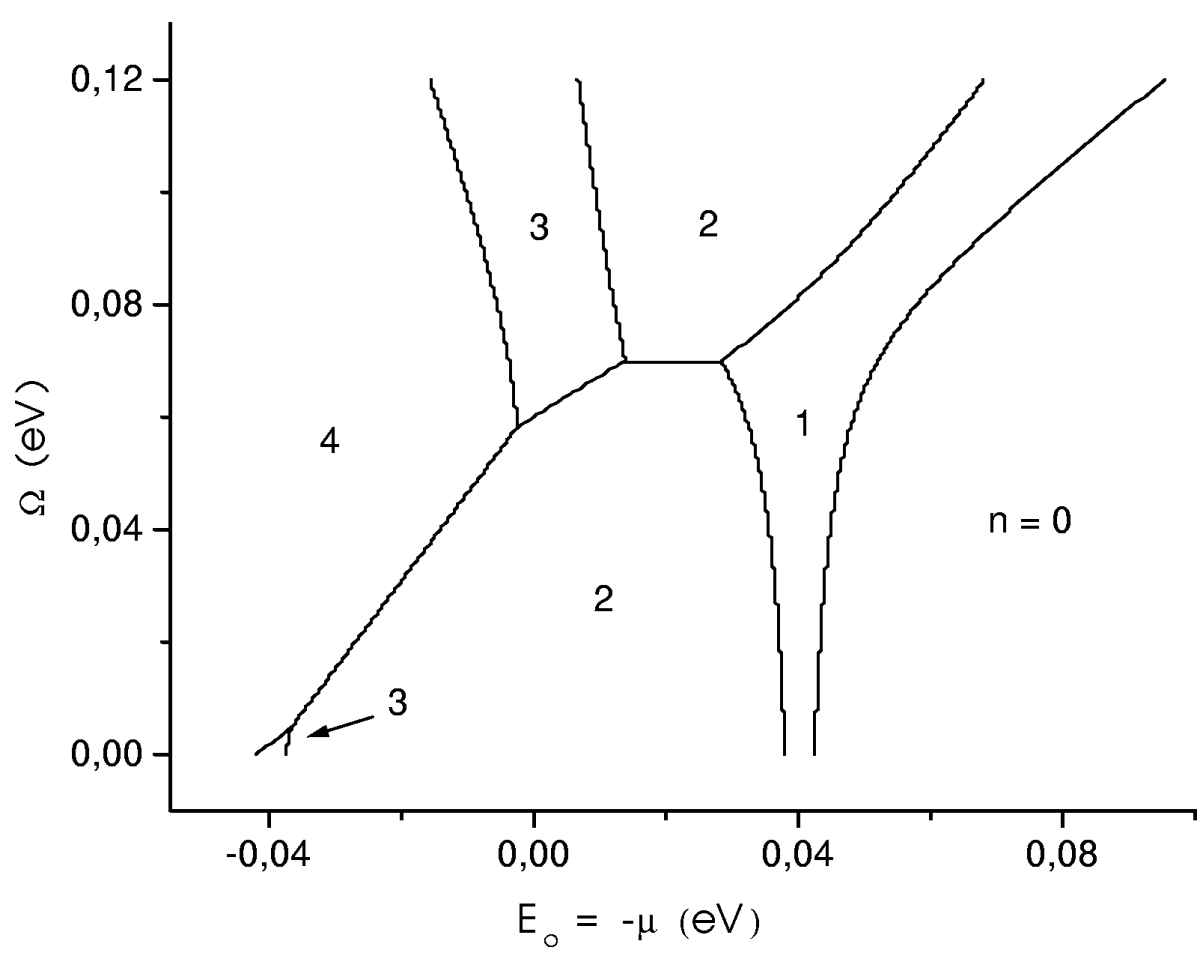

(b)

Figure 3. Ground state diagram in $\Omega-E_{0}$ plane: $g=0.08, \Delta_{0}=0.06, \Omega=0.1$; (a) $t=0$, (b) $t=0.05$ (all quantities are given in $\mathrm{eV}$ ). 
$1<n<2$
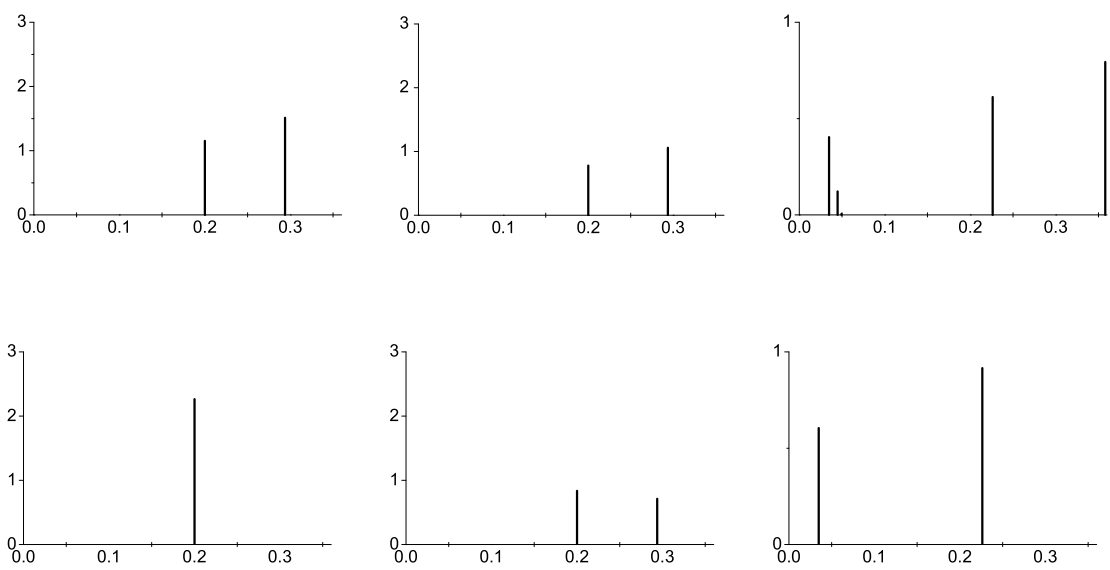

$n=1$
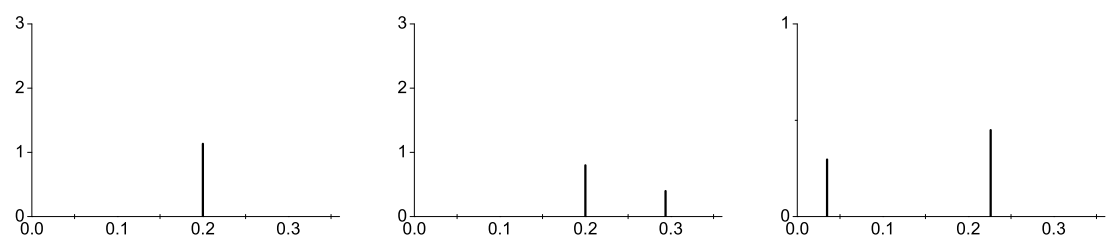

$0<n<1$
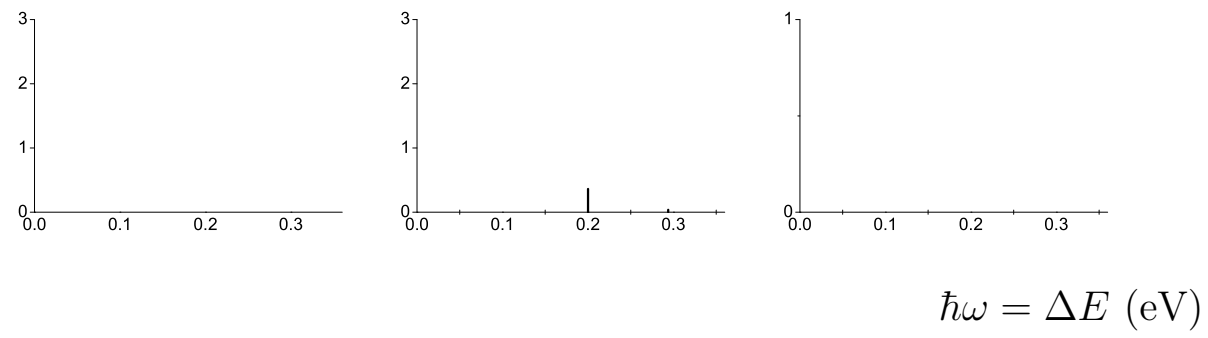

(a)

(b)

(c)

Figure 4. Frequency dependence of the dynamical conductivity: $g=0.08, \Delta_{0}=$ $0.06, \Omega=0.1$; (a) $t=0, T=0 \mathrm{~K}$; (b) $t=0, T=150 \mathrm{~K}$; (c) $t=0.05, T=0 \mathrm{~K}$. 

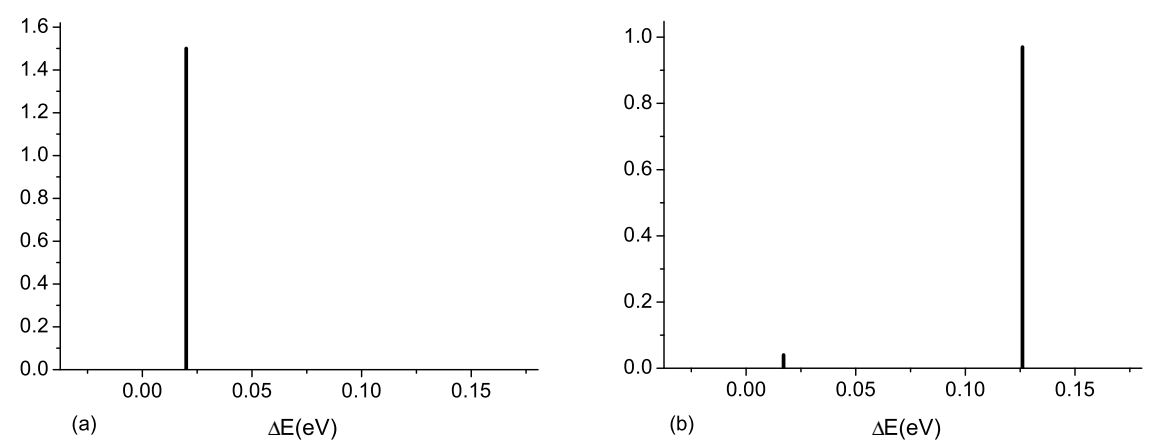

Figure 5. Frequency dependence of the dynamical conductivity: $g=0.06, \Delta_{0}=$ $0.013, \Omega=0.01$; (a) $t=0, \Delta E_{1}=0.02 \mathrm{eV}$; (b) $t=0.05, \Delta E_{1}=0.017 \mathrm{eV}$, $\Delta E_{2}=0.126 \mathrm{eV}, \sigma_{1}=4 \cdot 10^{-2}, \sigma_{2}=0.97, \frac{\left|\mu_{1}\right|^{2}}{\left|\mu_{2}\right|^{2}}=\frac{\sigma_{1} / \Delta E_{1}}{\sigma_{2} / \Delta E_{2}}=0.3$.

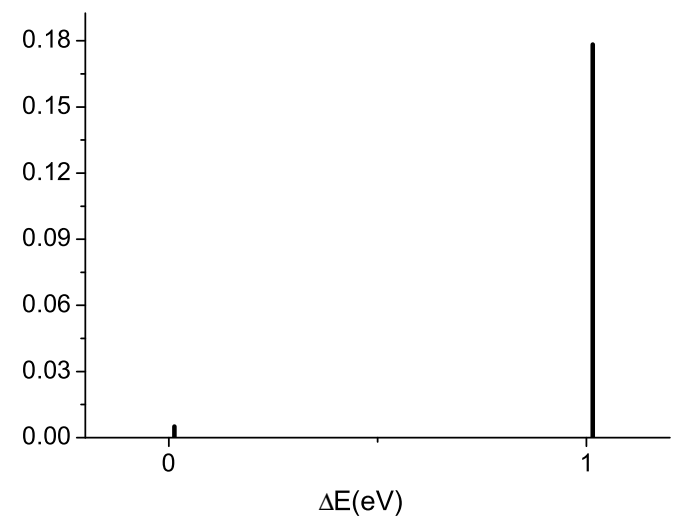

Figure 6. Frequency dependence of the dynamical conductivity; $g=-0.1, \Delta_{0}=$ 2.0, $\Omega=0.01, t=0.5, \Delta E_{1}=0.013 \mathrm{eV}, \Delta E_{2}=1.015 \mathrm{eV}, \sigma_{1}=4.16 \cdot 10^{-4}$, $\sigma_{2}=1.78 \cdot 10^{-1}, \frac{\left|\mu_{1}\right|^{2}}{\left|\mu_{2}\right|^{2}}=\frac{\sigma_{1} / \Delta E_{1}}{\sigma_{2} / \Delta E_{2}}=0.177$. 


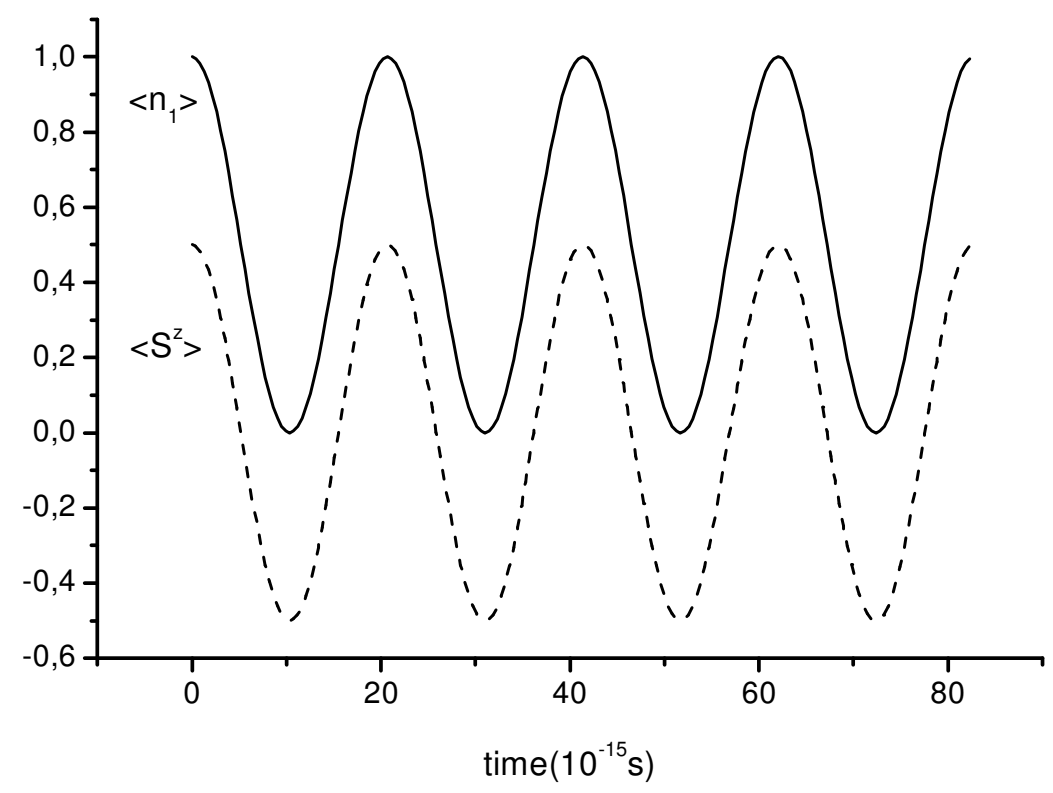

(a)

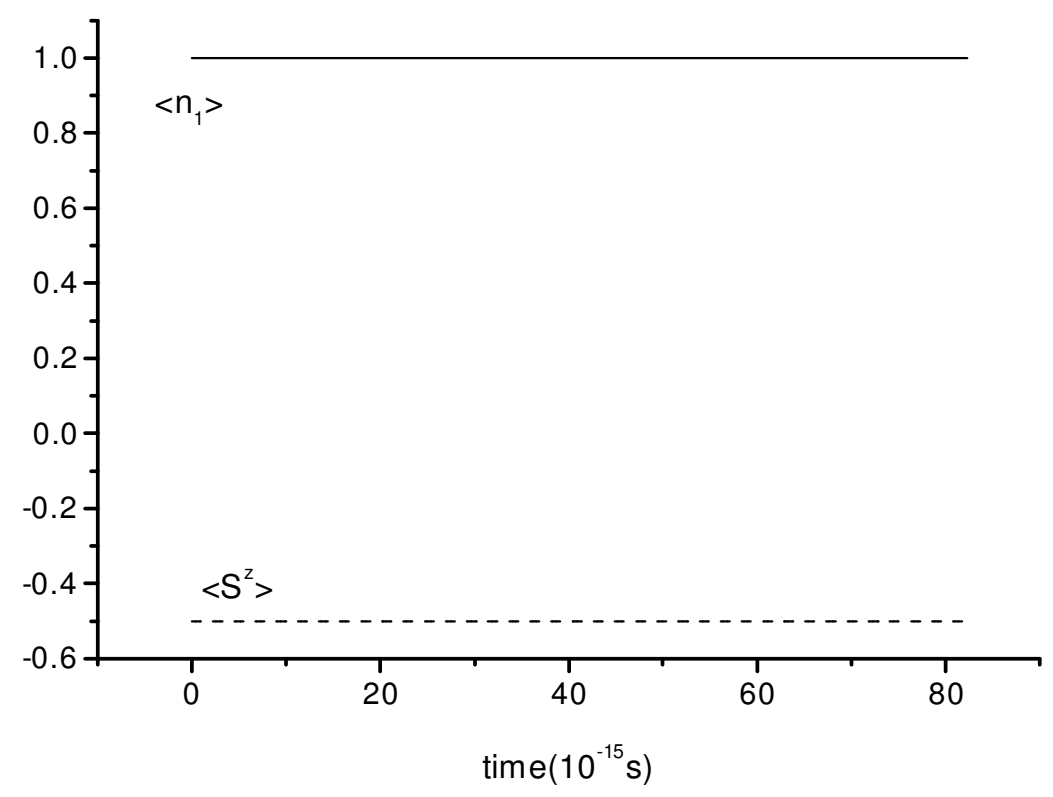

(b)

Figure 7. Time dependencies of mean values $\left\langle n_{1}\right\rangle$ and $\left\langle S^{z}\right\rangle$. Initial states (with one electron, $n=1)$ : (a) $|0100 ; 1 / 2\rangle$, (b) $|0100 ;-1 / 2\rangle$. $\left(g=0.08, \Delta_{0}=0.06\right.$, $\Omega=0.1, t=0)$. 


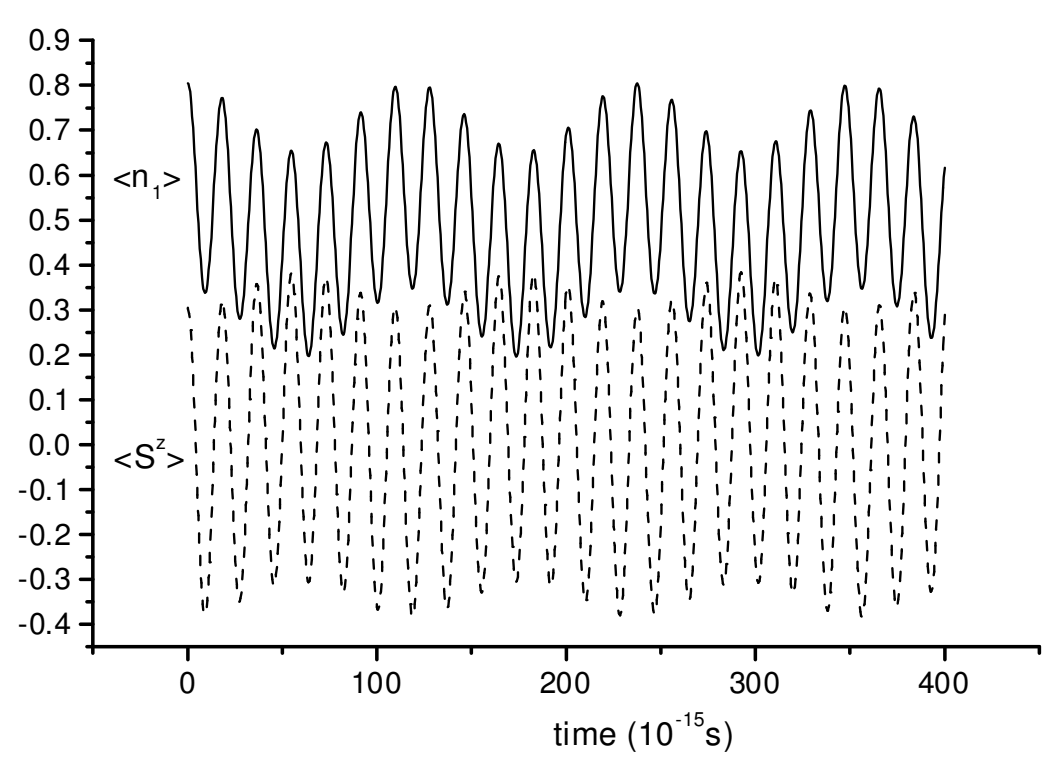

(a)

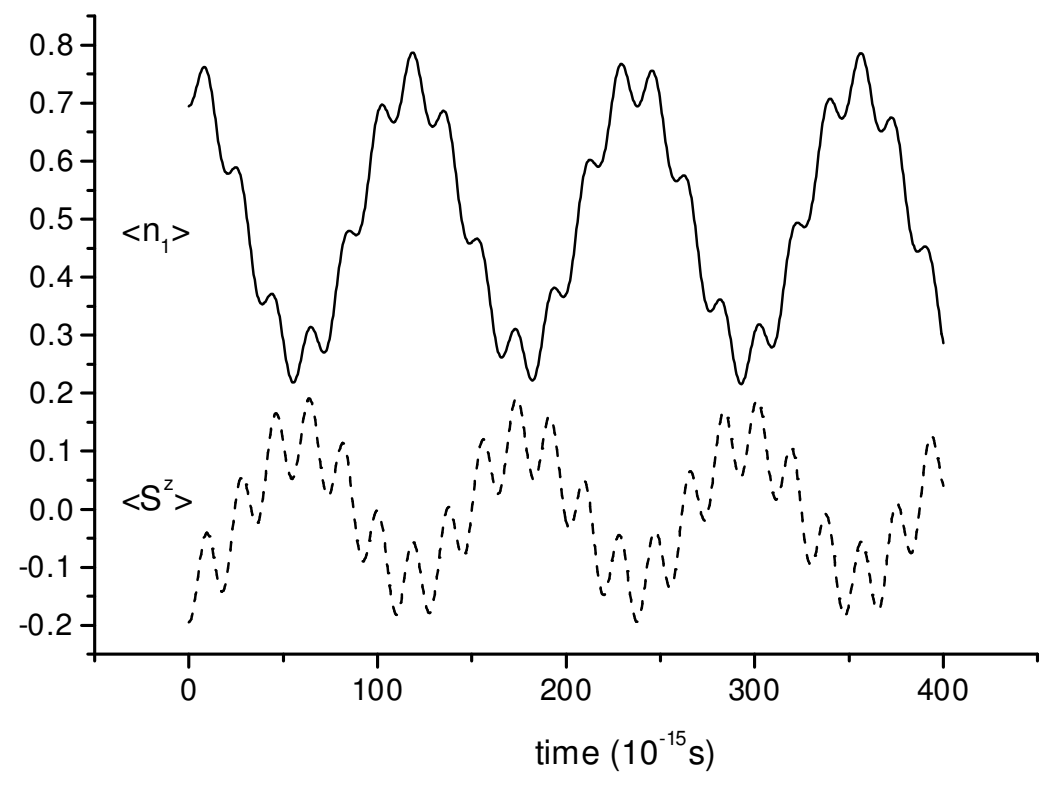

(b)

Figure 8. Time dependencies of mean values $\left\langle n_{1}\right\rangle$ and $\left\langle S^{z}\right\rangle$. Initial states (with one electron, $n=1)$ : (a) $|0100 ; 1 / 2\rangle$, (b) $|0100 ;-1 / 2\rangle ; g=0.08, \Delta_{0}=0.06$, $\Omega=0.1, t=0.05$. Transitions from ground state to excited states are taken into account (two harmonics). $\Delta E_{1}=0.226 \mathrm{eV}, \Delta E_{2}=0.035 \mathrm{eV}, T_{1}=18.2 \cdot 10^{-15} \mathrm{~s}$, $T_{2}=120 \cdot 10^{-15} \mathrm{~s}$. 


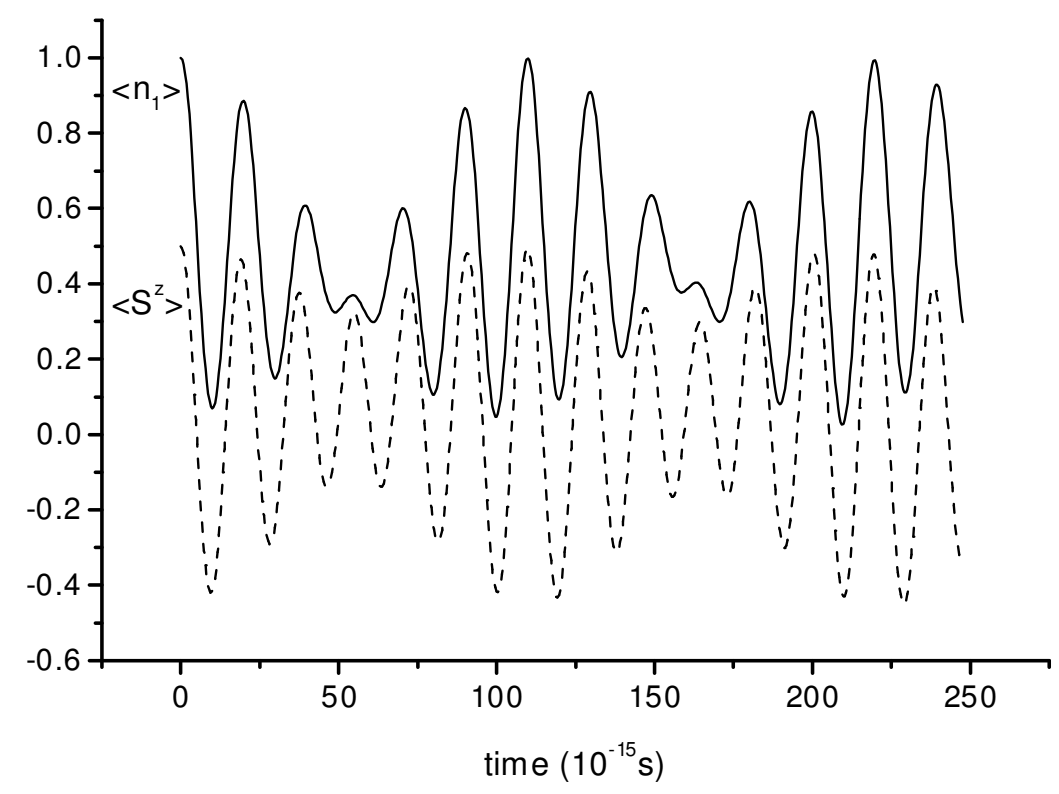

(a)

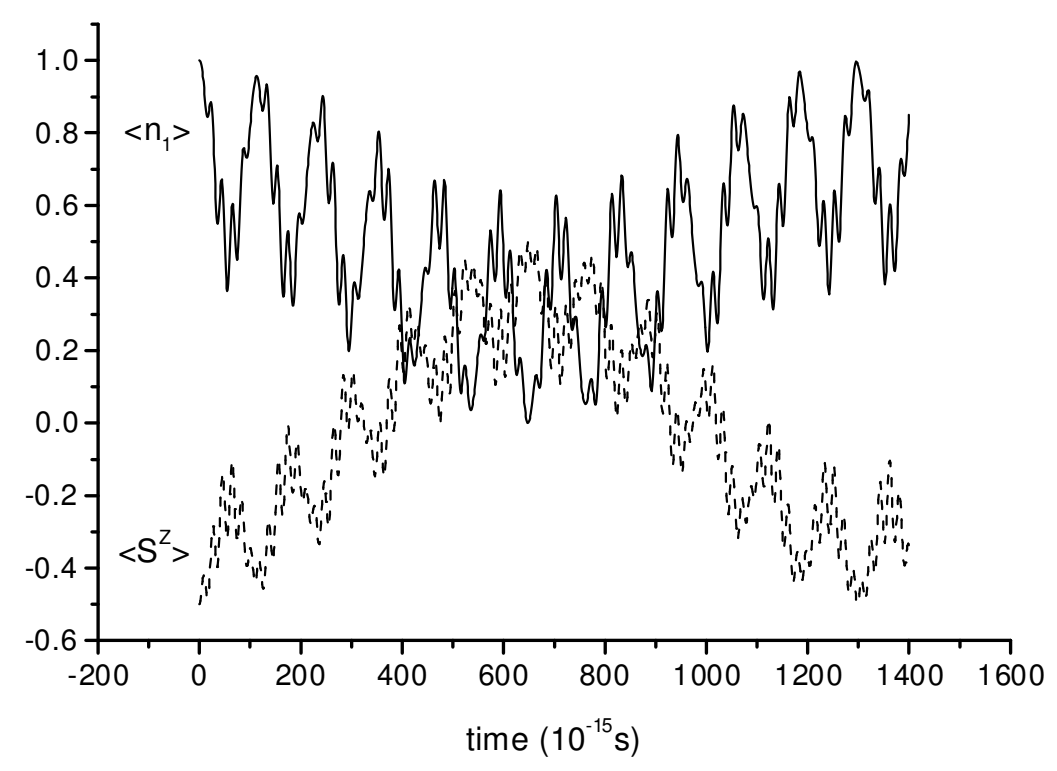

(b)

Figure 9. Time dependencies of mean values $\left\langle n_{1}\right\rangle$ and $\left\langle S^{z}\right\rangle$. Initial states (with one electron, $n=1$ ): (a) $|0100 ; 1 / 2\rangle$, (b) $|0100 ;-1 / 2\rangle ; g=0.08, \Delta_{0}=0.06$, $\Omega=0.1, t=0.05$. All possible transitions between states of the system with one electron on hydrogen bond are taken into account (four harmonics). 


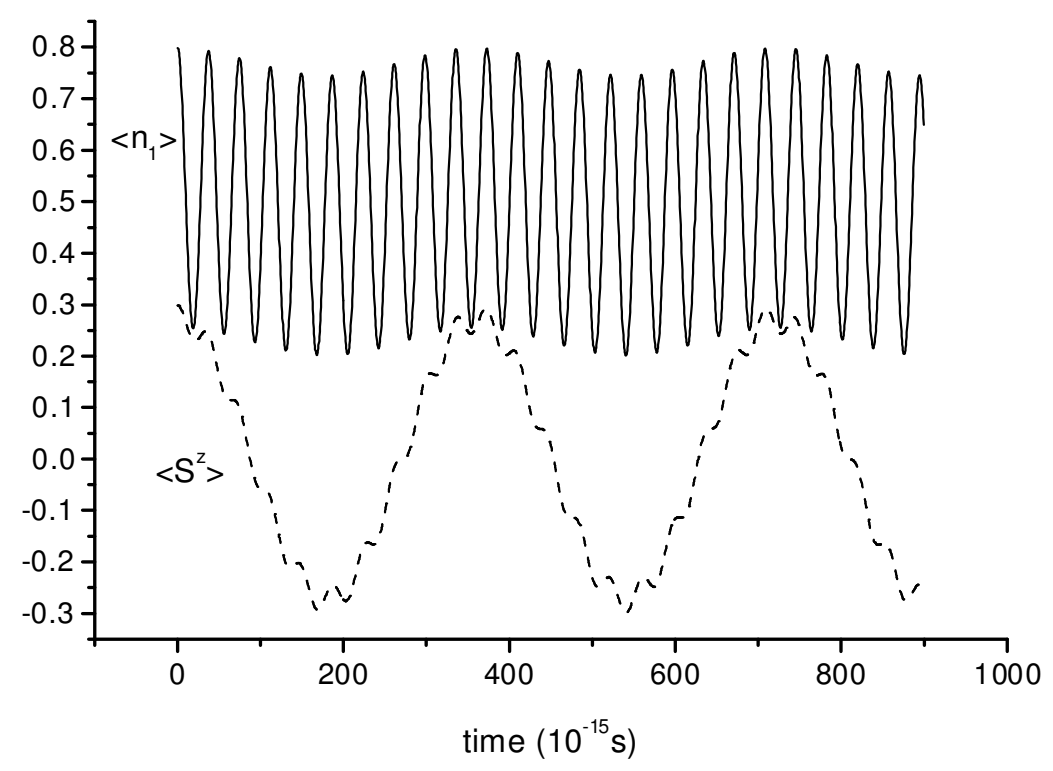

(a)

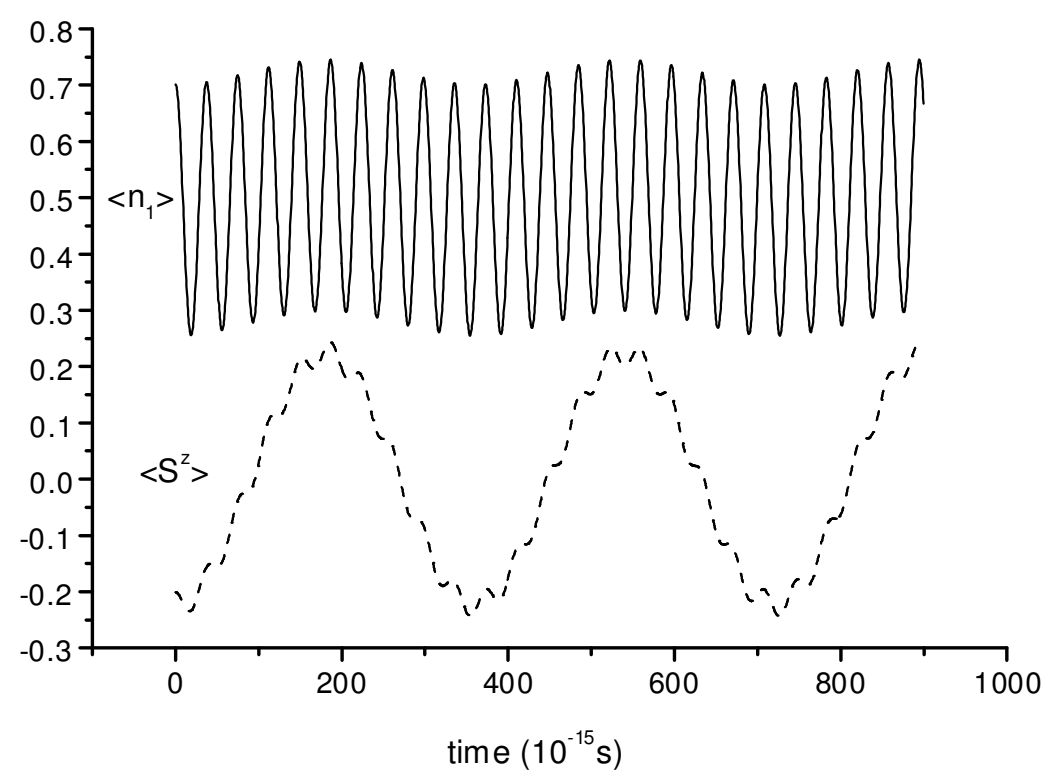

(b)

Figure 10. Time dependencies of mean values $\left\langle n_{1}\right\rangle$ and $\left\langle S^{z}\right\rangle$. Initial states (with one electron, $n=1$ ): (a) $|0100 ; 1 / 2\rangle$, (b) $|0100 ;-1 / 2\rangle ; g=-0.01, \Delta_{0}=1.0$, $\Omega=0.01, t=0.05$. Transitions from ground state to excited states are taken into account (two harmonics). $\Delta E_{1}=0.011 \mathrm{eV}, \Delta E_{2}=0.110 \mathrm{eV}, T_{1}=330 \cdot 10^{-15} \mathrm{~s}$, $T_{2}=33 \cdot 10^{-15} \mathrm{~s}$. 


\section{Conclusions}

The pseudospin-electron model is formulated in order to describe the protonelectron interaction and correlated charge transfer in complex with hydrogen bonds. Possible numerical values of the model parameters are analyzed.

It is shown that the energies of Slater-Takagi configurations depend not only on the proton number in each of $\mathrm{PO}_{4}$ group in the crystal as was conventionally considered. They are determined by the energies of proton transitions from one equilibrium state on hydrogen bonds into another, and their values to a great extent are caused by the net the influence of crystal surroundings.

There are two peaks (maxima) in the frequency dependence of the real part of conductivity, that is in accordance with the results of the time dynamics analysis. One of the peaks is connected mainly with the proton tunnelling on the bond while the others are partially determined by the proton-electron interaction. They shift to a higher frequency range if independent electron transfer is present $(t \neq 0)$ and in this case we obtain two groups of peaks; besides that, a new group of peaks appears at $t \neq 0$.

The intensities of peaks depend on temperature and on the mean number of electrons on the bond; the positions are determined by the proton-electron coupling constant as well as by the tunnelling and electron transfer parameter values.

The obtained results should be taken into account while interpreting the experimental data regarding frequency dispersion of conductivity in the hydrogen-bonded systems.

\section{Acknowledgement}

This work was partially supported by the Fundamental Research Fund of the Ministry of Ukraine for Science and Education (Project No. 02.07/00266).

\section{Appendix}

Calculations of energies of proton configurations near the $\mathrm{PO}_{4}$ groups in the $\mathrm{KH}_{2} \mathrm{PO}_{4}$ crystal were performed using quantum-chemical method AM1. Changes of the electron occupation of oxygen ions, forming a hydrogen bond were also examined at the proton displacement from one equilibrium position on the bond into another.

Elements of crystal KDP structure constructed by one, two or eight tetrahedron $\mathrm{PO}_{4}$, connected by hydrogen bonds were considered and energies of configurations with proton localization in different equilibrium positions on hydrogen bonds were analyzed. Configurations with two protons near each $\mathrm{PO}_{4}$ group are lower in energy. Proton displacement along the hydrogen bond from one group towards another leads to the formation of configurations with one and three protons. This state is energetically higher than the initial one. In the noninteracting tetrahedron model, the energy of creation of configurations with one and three protons (so-called Slater-Takagi configurations) is $\Delta E=2 w=E^{(1)}+E^{(3)}-2 E^{(2)}$. Here $E^{(n)}$ are the 
Table 1. Change of energy of clusters consisting of two tetrahedrons $\Delta E^{(2)}$ and clusters consisting of eight tetrahedrons $\Delta E^{(8)}$ at the proton displacement on the central bond from one equilibrium position into another, or energy of creation of configuration with one and three protons.

\begin{tabular}{|c|c|c|}
\hline configuration & $\Delta E^{(2)}, \mathrm{kcal} / \mathrm{mol}$ & $\Delta E^{(8)}, \mathrm{kcal} / \mathrm{mol}$ \\
\hline 1 & 1.53 & 0.99 \\
2 & 1.42 & 1.38 \\
3 & 1.31 & 1.645 \\
4 & 1.2 & 1.69 \\
5 & 1.23 & 5.97 \\
6 & 0.77 & 2.98 \\
7 & 1.25 & 5.75 \\
8 & 1.0 & 2.77 \\
9 & 0.82 & 8.91 \\
\hline
\end{tabular}

Table 2. Proton configurations of clusters consisting of two $\mathrm{PO}_{4}$ tetrahedrons. Large squares in pictures are the $\mathrm{PO}_{4}$ groups, black circles are the protons in equilibrium positions on the bond.

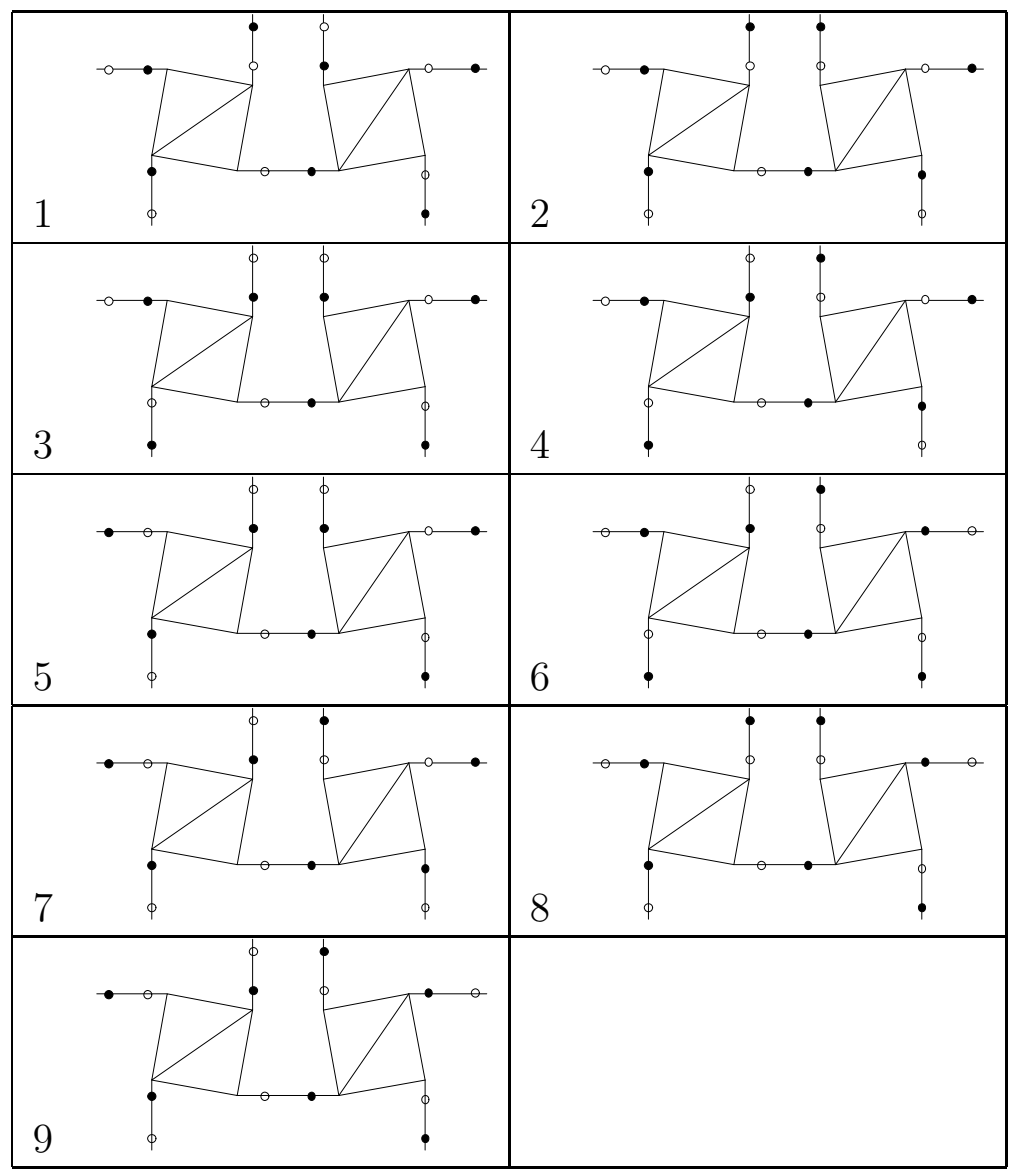



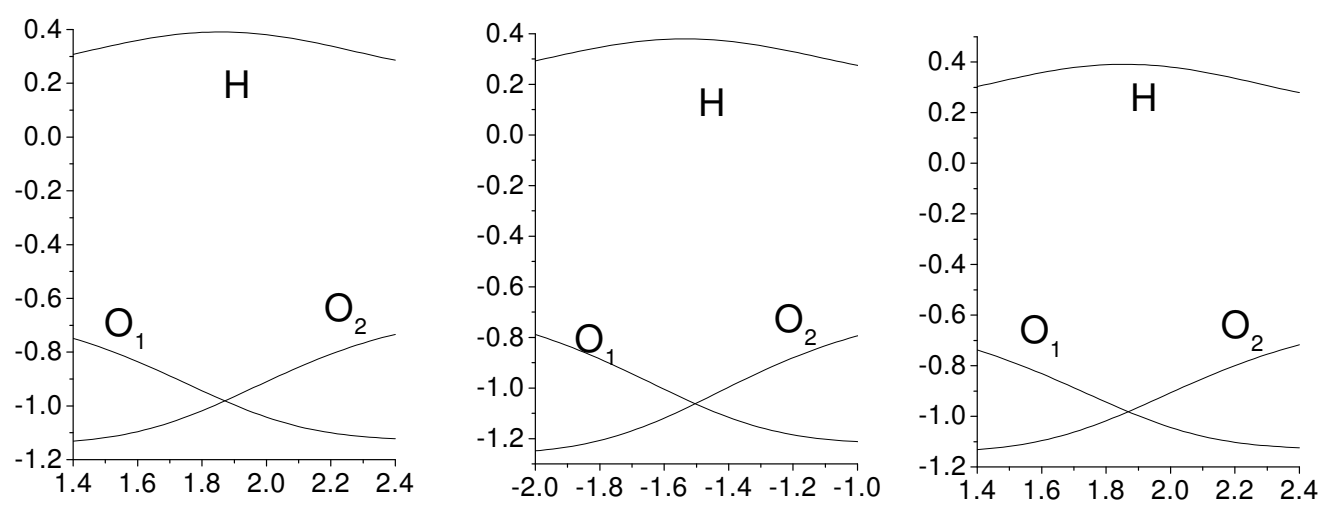

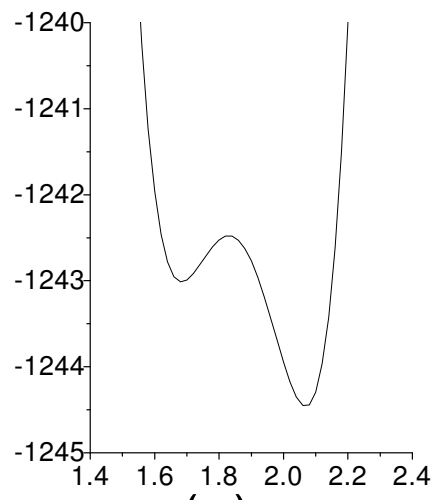

(a)

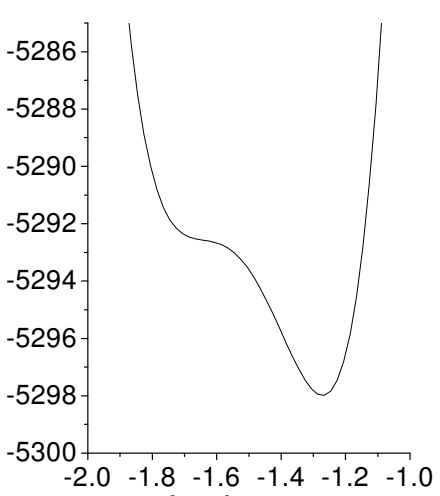

$\left(a^{\prime}\right)$

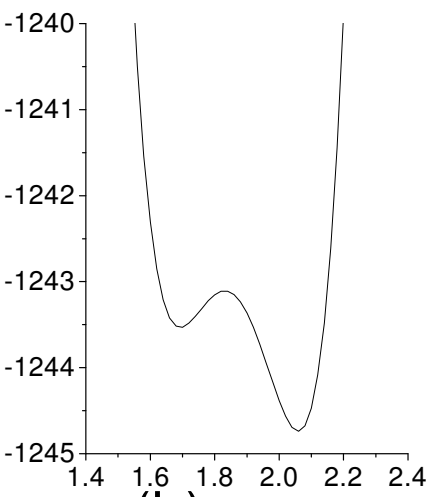

(b)
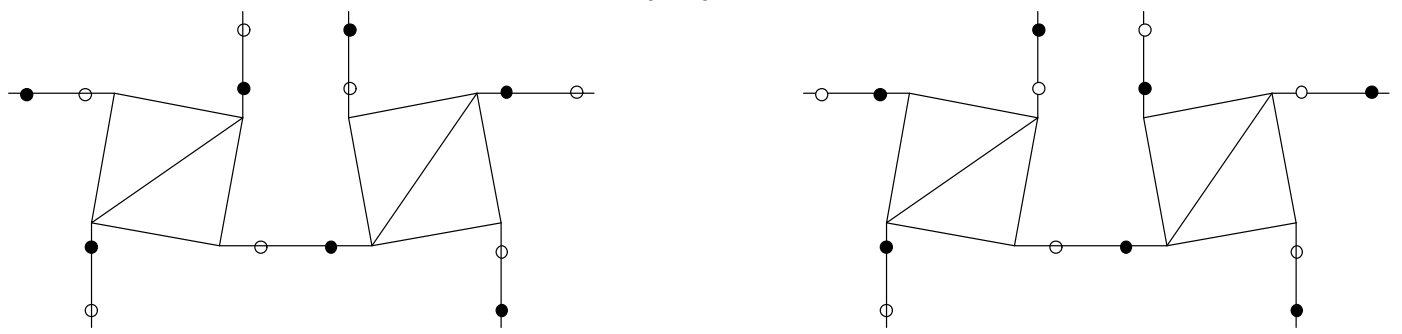

Figure 11. Adiabatic protons potentials and charges on hydrogen and oxygen ions, which forms a hydrogen bond, as functions of proton position on the bond for clusters presented in pictures; cluster consisting of eight tetrahedrons $\left(a^{\prime}\right)$ is constructed from the cluster (a) containing two $\mathrm{PO}_{4}$ tetrahedrons by adding to them the nearest neighbouring tetrahedrons connecting with the central tetrahedron by the hydrogen bond as in the KDP crystal. 
energies of $\mathrm{H}_{n} \mathrm{PO}_{4}$ configurations with $n$ protons in the near (to $\mathrm{PO}_{4}$ tetrahedron) equilibrium position for proton on the hydrogen bond and $(4-n)$ protons in the faroff positions. The following values of $E^{(n)}$ were obtained: $E^{(0)}=-460.18 \mathrm{kcal} / \mathrm{mol}$, $E^{(1)}=-503.79 \mathrm{kcal} / \mathrm{mol}, E^{(2)}=-547.01 \mathrm{kcal} / \mathrm{mol}, E^{(3)}=-589.92 \mathrm{kcal} / \mathrm{mol}$, $E^{(4)}=-631.79 \mathrm{kcal} / \mathrm{mol}$. This results in $\Delta E=2 w=0.31 \mathrm{kcal} / \mathrm{mol}$. The energy of creation of tetrahedron with one or three protons $(w)$ is equal $w=0.155 \mathrm{kcal} / \mathrm{mol}$ $\left(54.2 \mathrm{~cm}^{-1}\right)$, that is an order of magnitude lower than the data known in literature (see, for example $[16,17]$ ). Consideration of interactions not only in $\mathrm{H}_{n} \mathrm{PO}_{4}$ groups but between all parts of the cluster consisting of a few tetrahedrons, gives the energy values much closer to those known in the literature. The change of cluster energy obtained here at proton displacement on the central bond from one equilibrium position into another is $\Delta E=2 w$. In other words, the energy of creation of a configuration with one and three protons depends essentially on the initial configuration with two protons near each $\mathrm{PO}_{4}$ group. Thus, it is to a great extent determined by the field of crystal surroundings for the bond, along which the proton is displaced. Hence, the energies of Slater-Takagi configurations depend not only on proton number on each $\mathrm{PO}_{4}$ group in KDP-type crystal. Calculated values of $\Delta E$ for the cluster consisting of two tetrahedrons $\left(\Delta E^{(2)}\right)$ and of eight tetrahedrons $\left(\Delta E^{(8)}\right)$ are presented in table 1. Initial cluster configurations (for cluster of two tetrahedrons) are presented in table 2. In cluster N9 consisting of eight tetrahedrons, as well as in the corresponding cluster consisting of two tetrahedrons, all protons are located near the upper oxygen atoms at each $\mathrm{PO}_{4}$ tetrahedron. The rest clusters consisting of eight tetrahedrons are constructed from the cluster N9 by the transition to other proton configurations on two central groups, shown in table 2. In clusters consisting of eight tetrahedrons, the crystal field is taken more accurately. For this case, the mean value of energy is $\Delta E^{(8)}=2 w=3.565 \mathrm{kcal} / \mathrm{mol}=1247 \mathrm{~cm}^{-1}$. So, in this case $w=623 \mathrm{~cm}^{-1}$. It should be noted that taking value $w=840 \mathrm{~K}\left(584 \mathrm{~cm}^{-1}\right)$ a variety of thermodynamical properties of KDP and DKDP crystals were described satisfactorily within the same approach (see $[16,17]$ ).

The results of calculation of adiabatic potential for proton on the bond for some proton configurations are presented in figure 11. Dependences of electron occupation of the left and the right oxygens forming hydrogen bond on the proton position on the bond are also presented there. In the cluster consisting of eight tetrahedrons $\left(\mathrm{a}^{\prime}\right)$ as well as in the cluster consisting of two tetrahedrons (a) all protons are located near the upper oxygen atoms at each $\mathrm{PO}_{4}$ tetrahedron. In the cluster consisting of two tetrahedrons presented in figure 11 (b), the so-called lateral Slater configurations are realized. As one can see, proton jump from one minimum of adiabatic potential on the bond into another is accompanied by the change of oxygen occupation $\Delta n=0.19$ which is practically independent of the initial proton configuration. 


\section{References}

1. Stasyuk I.V., Ivankiv O.L., Pavlenko N.I. // J. Phys. Studies, 1997, vol. 1. p. 418.

2. Gaididei Y., Flytzanis N., Yanovitskii O. // Solid State Ionics, 1995, vol. 77, p. 20.

3. Okaniwa K., Okamoto H., Mitani T., Toriumi K., Yamashita M. // J. Phys. Soc. Japan, 1991, vol. 60, No. 3, p. 997.

4. Mitani T., Kitagawa H., Okamoto H., Nakasuji K., Toyota J., Yamashita M. // Mol. Cryst. Liq. Cryst., 1992, vol. 216, p. 73.

5. Takeda S., Chihara H., Inabe T., Mitani T., Maruyama Y. // Mol. Cryst. Liq. Cryst., 1992, vol. 216, p. 235.

6. Nakasuji K., Sugiura K., Toyoda J., Morita Y., Okamoto H., Okaniwa K., Mitani T. // Mol. Cryst. Liq. Cryst., 1992, vol. 216, p. 213.

7. Inabe T., Okaniwa K., Okamoto H., Mitani T., Maruyama Y., Takeda S. // Mol. Cryst. Liq. Cryst., 1992, vol. 216, p. 229.

8. Stasyuk I.V., Sizonenko Yu.V., Stetsiv R.Ya. // J. Phys. Studies, 1998, vol. 2, No. 4, p. 463.

9. Hillenbrand E.A., Scheiner S. // J. Amer. Chem. Soc., 1984, vol. 106, No. 21, p. 6266.

10. Matsushita E. // Phys. Rev. B, 1995, vol. 51, No. 24, p. 17332.

11. Mittal R., Howard I.A. // Phys. Rev. B, 1996, vol. 53, No. 21, p. 14171.

12. Howard I.A., Mittal R. // Phys. Rev. B, 1998, vol. 57, No. 1, p. 45.

13. Müller K.A. // Z. Phys. B, 1990, vol. 80, No. 2, p. 193.

14. Kubo R. // J. Phys. Soc. Jpn., 1957, vol. 12, p. 570.

15. Mackowiak M. // J. Mol. Struct., 1989, vol. 192, p. 189.

16. Stasyuk I.V., Levitskii R.R., Moina A.P. // Phys. Rev. B, 1999, vol. 59. No. 13, p. 8530 .

17. Stasyuk I.V., Levitskii R.R., Zachek I.R., Moina A.P. // Phys. Rev. B, 2000, vol. 62, No. 10, p. 6198. 


\section{Динаміка переносу заряду вздовж водневого зв'язку}

\section{І.В.Стасюк, Р.Я.Стеців, Ю.В.Сизоненко}

Інститут фізики конденсованих систем НАН України, 79011 Львів, вул. Свєнціцького, 1

Отримано 13 вересня 2002 р.

Запропоновна псевдоспін-електронна модель для опису скорельованого протон-електронного переносу заряду на комплексі з водневим зв'язком. Отримано енергетичний спектр моделі. Побудовано фазову діаграму основного стану. Розраховано частотну залежність дійсної частини динамічної провідності. Досліджена часова еволюція протонного і електронного транспорту вздовж водневого зв'язку. Розв'язані рівняння руху для компонент матриці густини, отримана часова залежність середньої заселеності протонних позицій і електронних станів. Встановлені умови, при яких рух протонів і електронів $\epsilon$ скорельований.

Ключові слова: псевдоспін-електронна модель, протон-електронний перенос, водневий зв'язок, провідність

PACS: $72.60 .+g, 36.40 . \mathrm{C}$ 